Supporting Information

\title{
Stable Hydrogel Electrolytes for Flexible and Submarine-Use Zn-Ion Batteries
}

Baojun Wang ${ }^{a}$, Jianmin Li ${ }^{a}$, Chengyi Hou ${ }^{a}$, Qinghong Zhang ${ }^{b}$, Yaogang Li ${ }^{b *}$, and

Hongzhi Wang ${ }^{a *}$

${ }^{\text {a }}$ State Key Laboratory for Modification of Chemical Fibers and Polymer Materials,

College of Materials Science and Engineering, Donghua University, Shanghai

201620, People's Republic of China.

${ }^{\mathrm{b}}$ Engineering Research Center of Advanced Glasses Manufacturing Technology,

Ministry of Education, Donghua University, Shanghai 201620, People's Republic of

China.

* Corresponding author.

E-mail addresses: yaogang_li@dhu.edu.cn (Y. G. Li), wanghz@ dhu.edu.cn (H. Z.

Wang) 

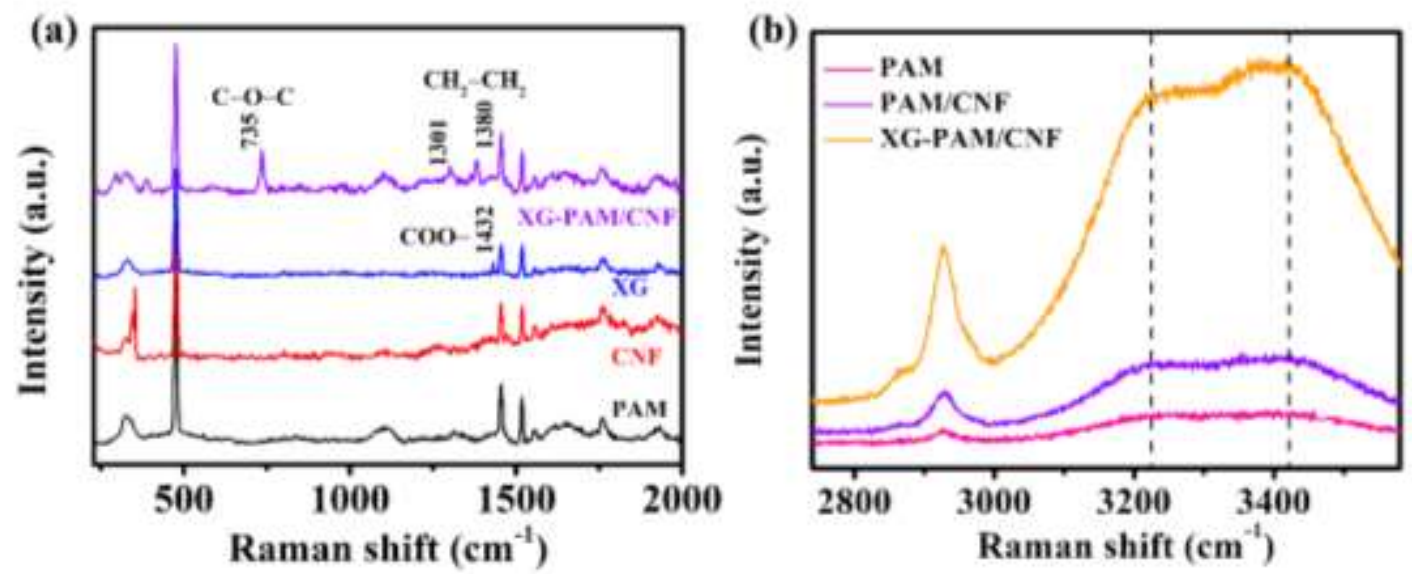

Figure S1. (a) The Raman spectra of each component of PAM, CNF, XG, and XGPAM/CNF hydrogels, (b) The Raman spectra of PAM, PAM/CNF, XG-PAM/CNF hydrogels in specific ranges.

The Raman spectra in Figure S1a confirms the graft polymerization of XG-PAM, with the formation of ether link and carbon-carbon single bond. Besides, as revealed in Figure S1b, the water vibration bands of PAM, PAM/CNF and XG-PAM/CNF hydrogels are all located at $3220 \mathrm{~cm}^{-1}$ and $3420 \mathrm{~cm}^{-1}$, which represent the intermolecular hydrogen bonds and the $\mathrm{OH}$-stretching, respectively. ${ }^{1}$ Therefore, it reflects the hydrogen bonding interactions are formed in polymer networks. Furthermore, the $\mathrm{OH}$-stretching vibration band of water is elevated with the addition of $\mathrm{CNF}$ and $\mathrm{XG}$ components into pure PAM hydrogel, indicating stronger interactions. 

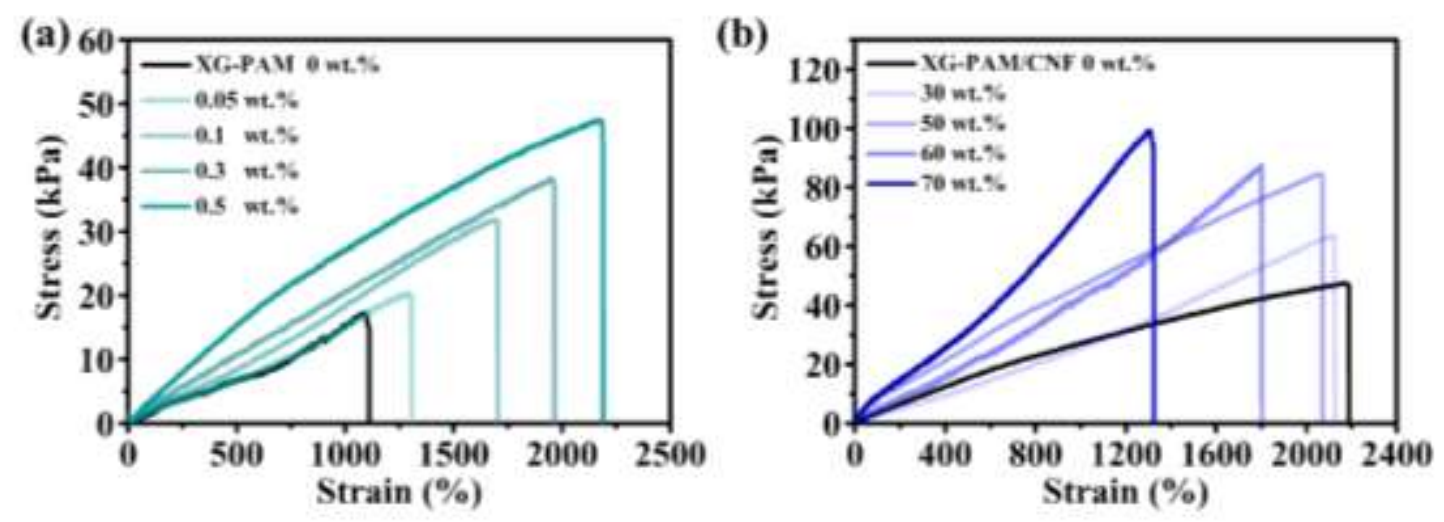

Figure S2. The stress-strain curves of different mass ratio between XG, PAM and CNF.

(a) Stress-strain curves of XG-PAM hydrogel with different XG mass ratio, (b) Stressstrain curves of XG-PAM/CNF hydrogel with different CNF mass ratio.

Firstly, we adjusted the mass ratio of XG to PAM, and found that the mechanical properties of XG-PAM were improved with the increasing contents of XG (Figure S2a). It is worth noting that suitable XG content results in hydrogels, however, when the mass ratio of XG to PAM was higher than $0.5 \mathrm{wt} . \%$, the sol solution with mixing CNF could be very viscous and hard to form the proper hydrogel, because of the thickener property of XG. Hence, we selected the mass ratio of XG to PAM was 0.5 wt.\%. Secondly, we adjusted the mass ratio of CNF to XG-PAM, and found that the mechanical strength of $\mathrm{XG}-\mathrm{PAM} / \mathrm{CNF}$ were enhanced while the elasticity of XG-PAM/CNF were weakened with the increasing contents of CNF (Figure $\mathbf{S 2 b}$ ). Therefore, we selected the mass ratio of CNF to XG-PAM was 50 wt.\% to be the optimum. Finally, the optimal case could be 0.5 wt. $\%$ XG and 50 wt. $\%$ CNF. 


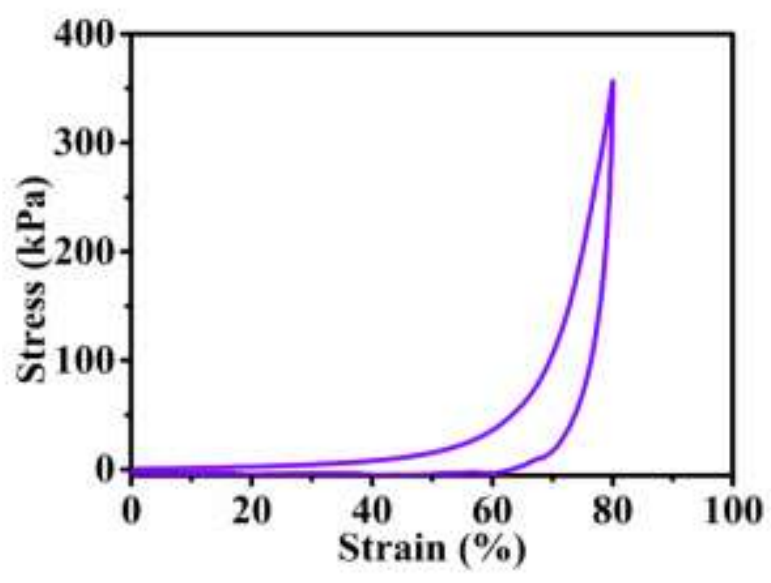

Figure S3. Loading-unloading test of the XG-PAM/CNF hydrogel under a compression of $80 \%$.

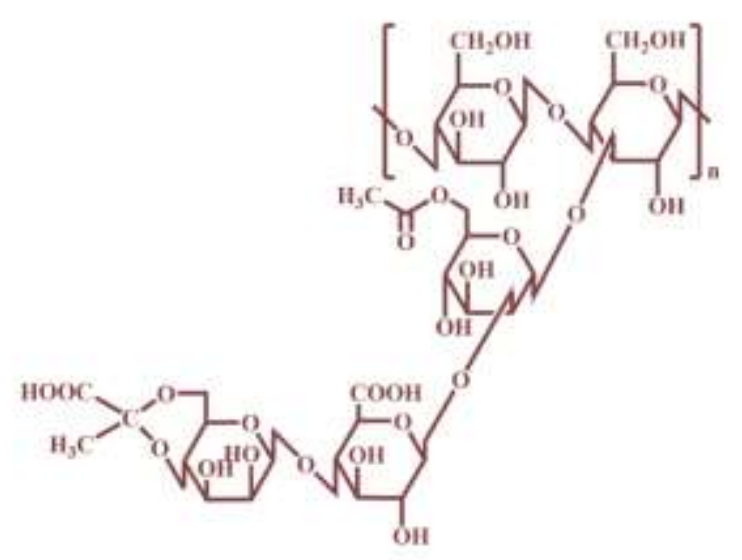

Figure S4. Chemical structure of xanthan gum. 


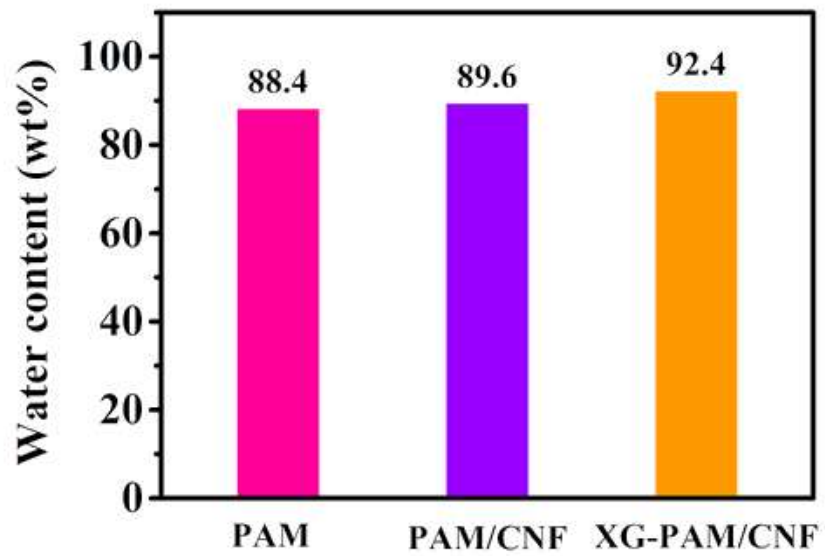

Figure S5. Water content of PAM, PAM/CNF and XG-PAM/CNF hydrogel.

The water content of the hydrogels was measured by the weight change in drying environment of vacuum oven at $120^{\circ} \mathrm{C}$. The columned hydrogels with the same volume and shape were mildly wiped the water off the surfaces by using a tissue before weighting. The water content is defined as the ratio in percentage between the weight of water in the hydrogels to the total weight of the hydrogels. As revealed in Figure S5, the XG-PAM/CNF hydrogel contained 92.4 wt.\% water content, which was improved after introducing XG and CNF in pure PAM. 


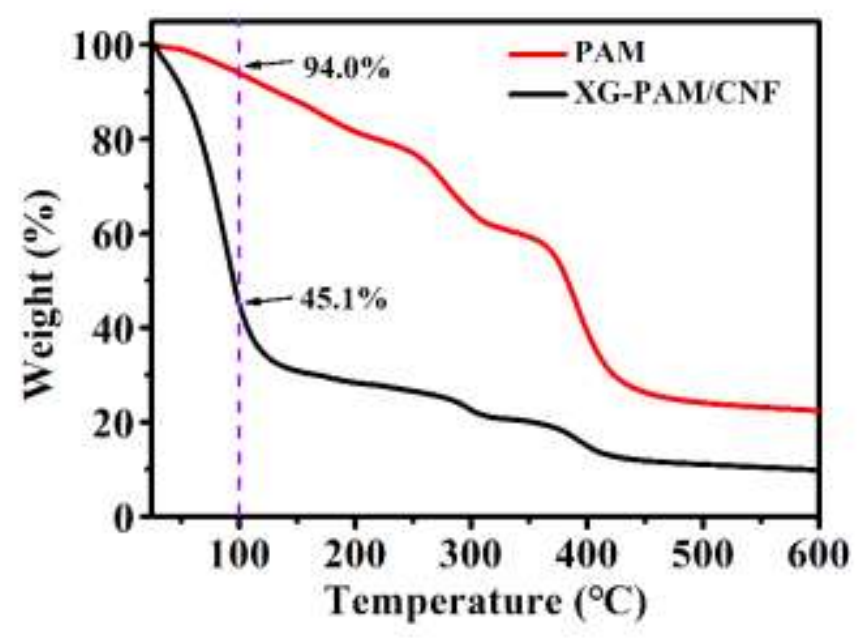

Figure S6. TG curves of PAM and XG-PAM/CNF hydrogel electrolyte with the temperature ranging from 30 to $600{ }^{\circ} \mathrm{C}$.

The TG curves of PAM and XG-PAM/CNF were obtained at heating rate of $10{ }^{\circ} \mathrm{C} / \mathrm{min}$ in $\mathrm{N}_{2}$ atmosphere are shown in Figure S6. It was clear that as compared to PAM, the XG-PAM/CNF remains more thermally stable. 


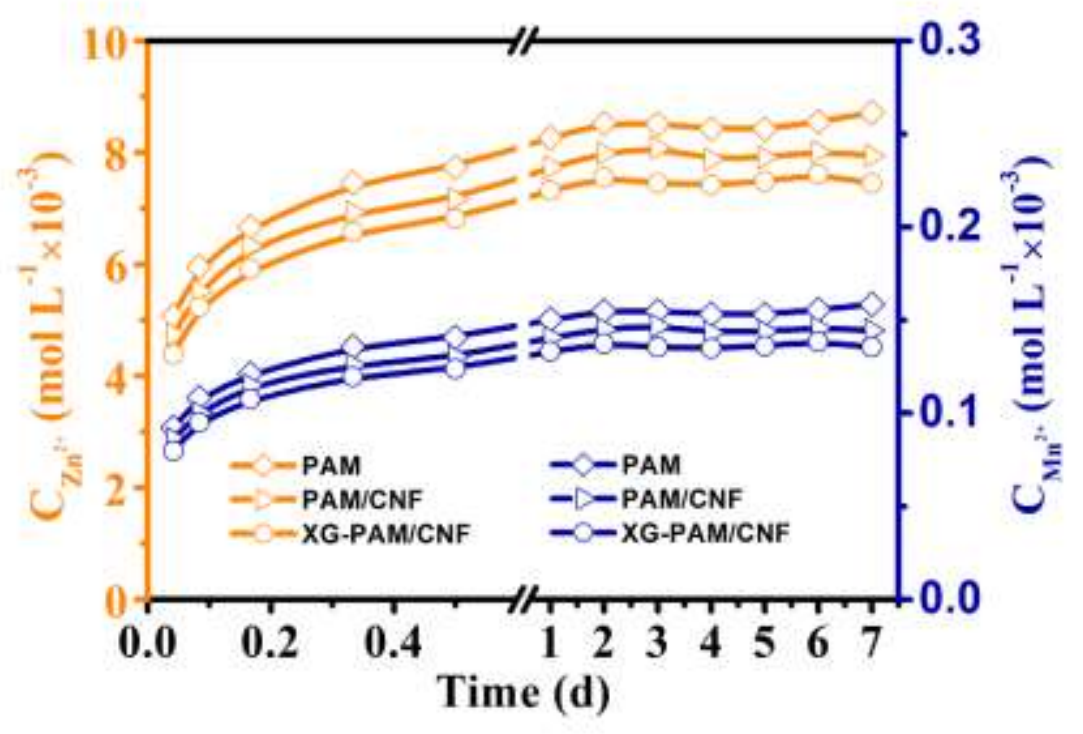

Figure S7. Inductively coupled plasma (ICP) analysis of $\mathrm{Zn}^{2+}$ and $\mathrm{Mn}^{2+}$ ions concentration in deionized water during 7 days. The tested water was soaked with PAM, PAM/CNF and XG-PAM/CNF hydrogel electrolyte.
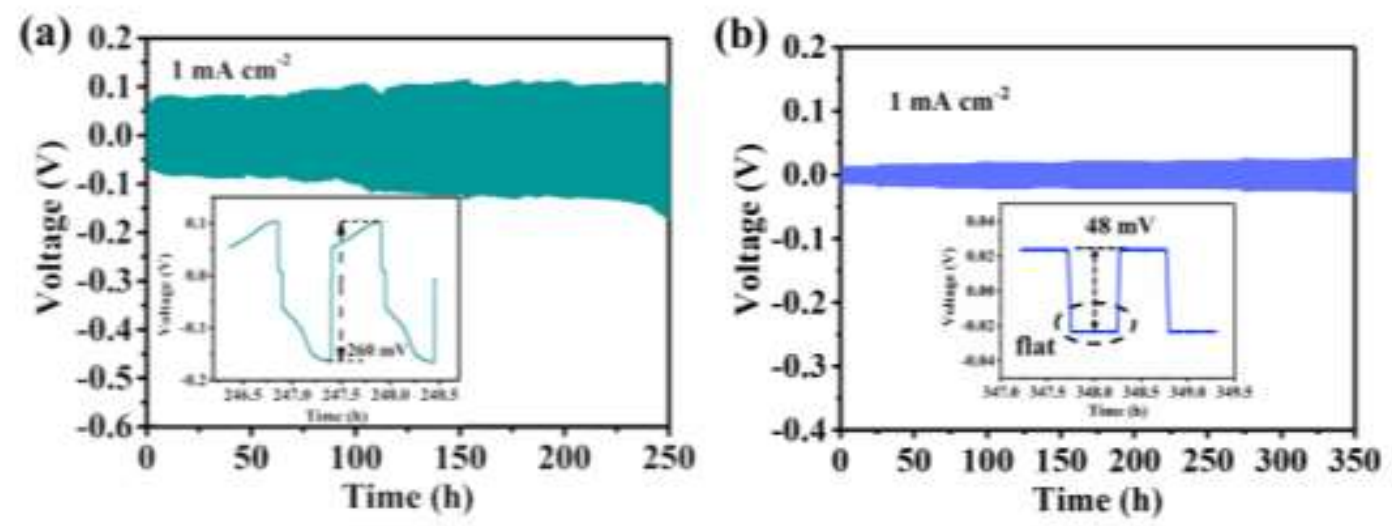

Figure S8. Galvanostatic cycling of symmetric $\mathrm{Zn} / / \mathrm{Zn}$ cells at a current density of 1 $\mathrm{mA} \mathrm{cm} \mathrm{cm}^{-2}$ and an areal capacity of $0.5 \mathrm{mAh} \mathrm{cm}^{-2}$ in the (a) PAM hydrogel electrolyte and (b) XG-PAM/CNF hydrogel electrolyte. 
(a)

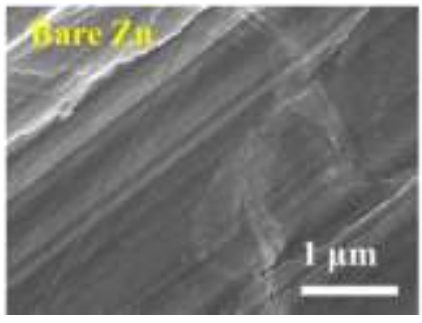

(b)

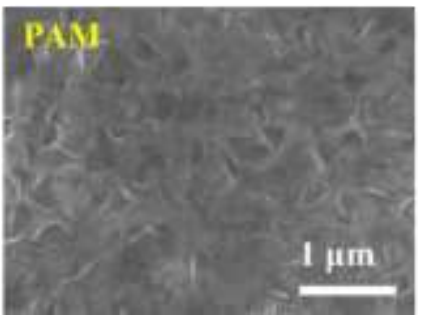

(c) XGPAM/CNF

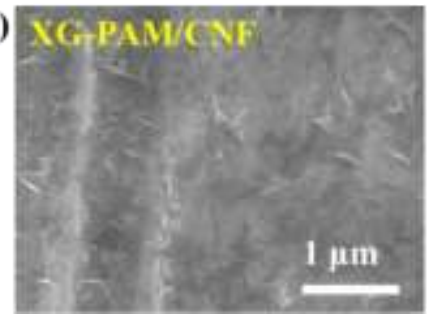

Figure S9. SEM images of surfaces of (a) bare polished $\mathrm{Zn}$ foil, (b) $\mathrm{Zn}$ foil cycled in

PAM hydrogel electrolyte, and (c) Zn foil cycled in XG-PAM/CNF hydrogel electrolyte.
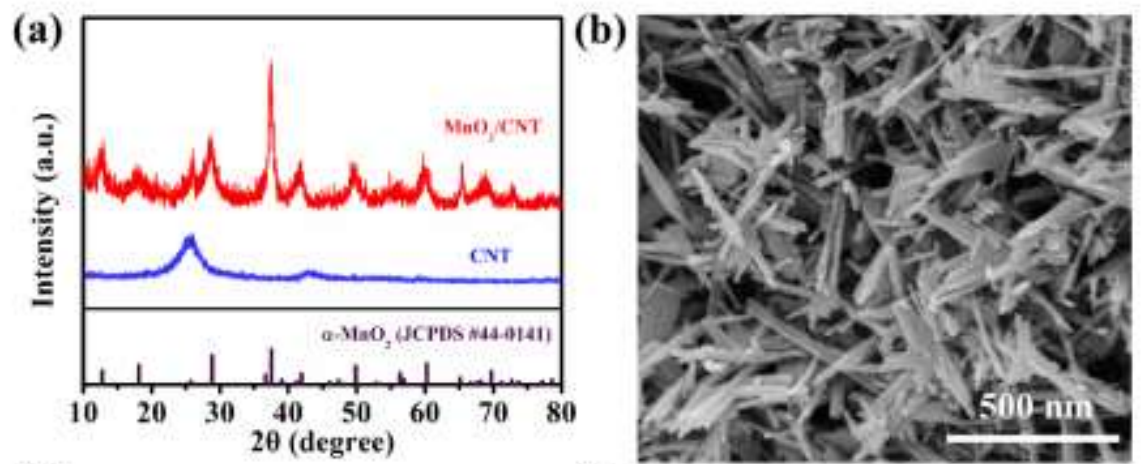

(c)

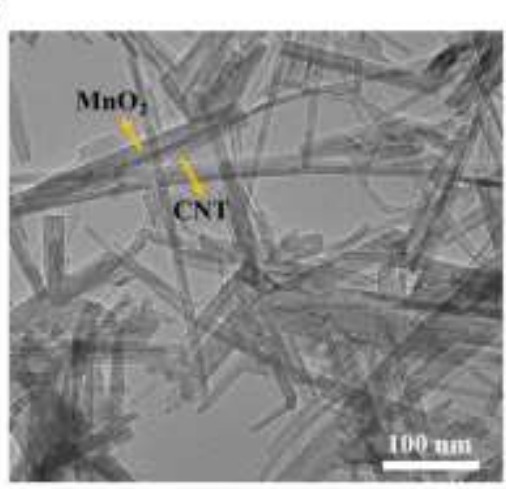

(d)

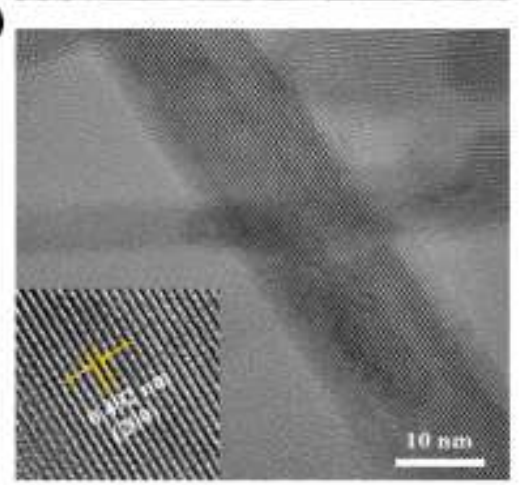

Figure S10. (a) XRD patterns of the $\mathrm{MnO}_{2} / \mathrm{CNT}$ composites and CNT, (b) SEM image of $\mathrm{MnO}_{2} / \mathrm{CNT}$ composites, TEM (c) and HRTEM (d) images of the $\mathrm{MnO}_{2} / \mathrm{CNT}$ composites. 

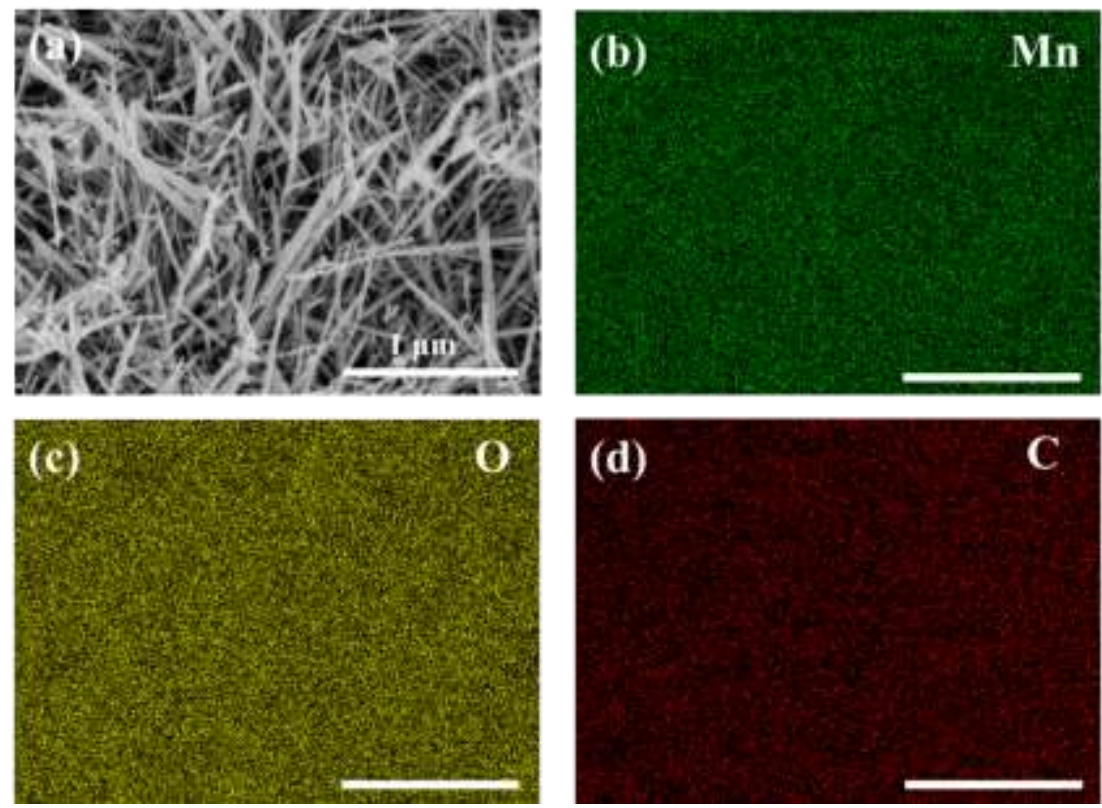

Figure S11. SEM image and EDS elemental mapping of $\mathrm{MnO}_{2} / \mathrm{CNT}$ nanocomposites.

(a)

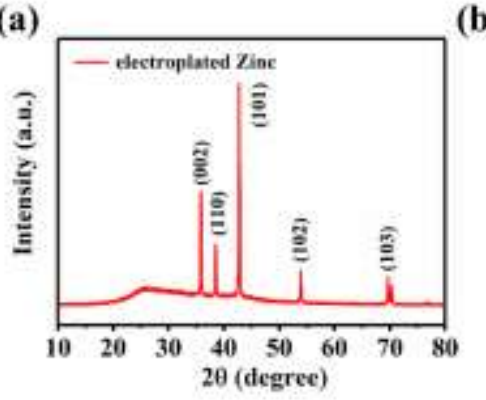

(b)

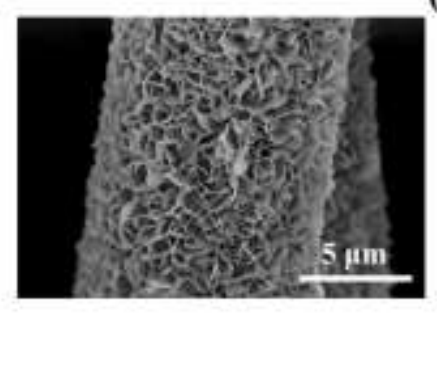

(c)

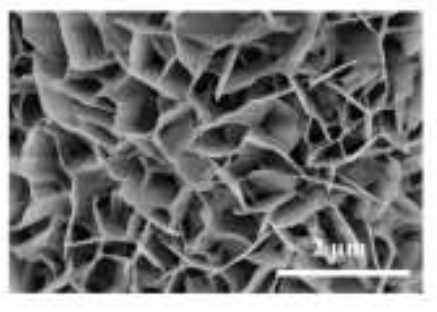

Figure S12. (a) XRD pattern of the electroplated zinc, (b, c) SEM images of zinc anode. 
(a)

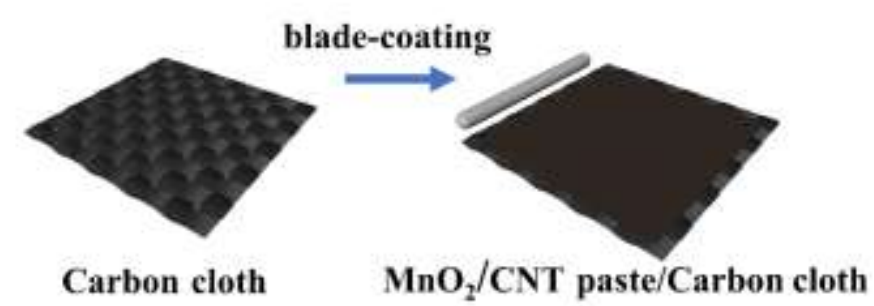

(b)

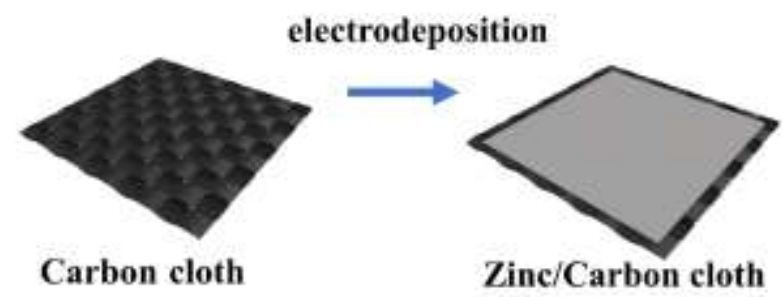

Figure S13. Schematic illustration of the preparation of flexible $\alpha-\mathrm{MnO}_{2} / \mathrm{CNT}$ cathode and zinc anode. 


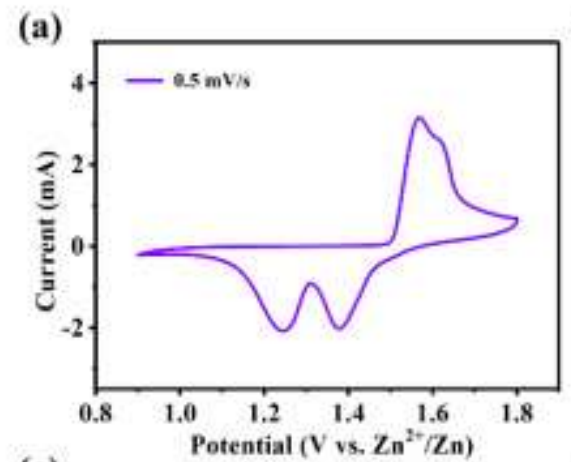

(b)
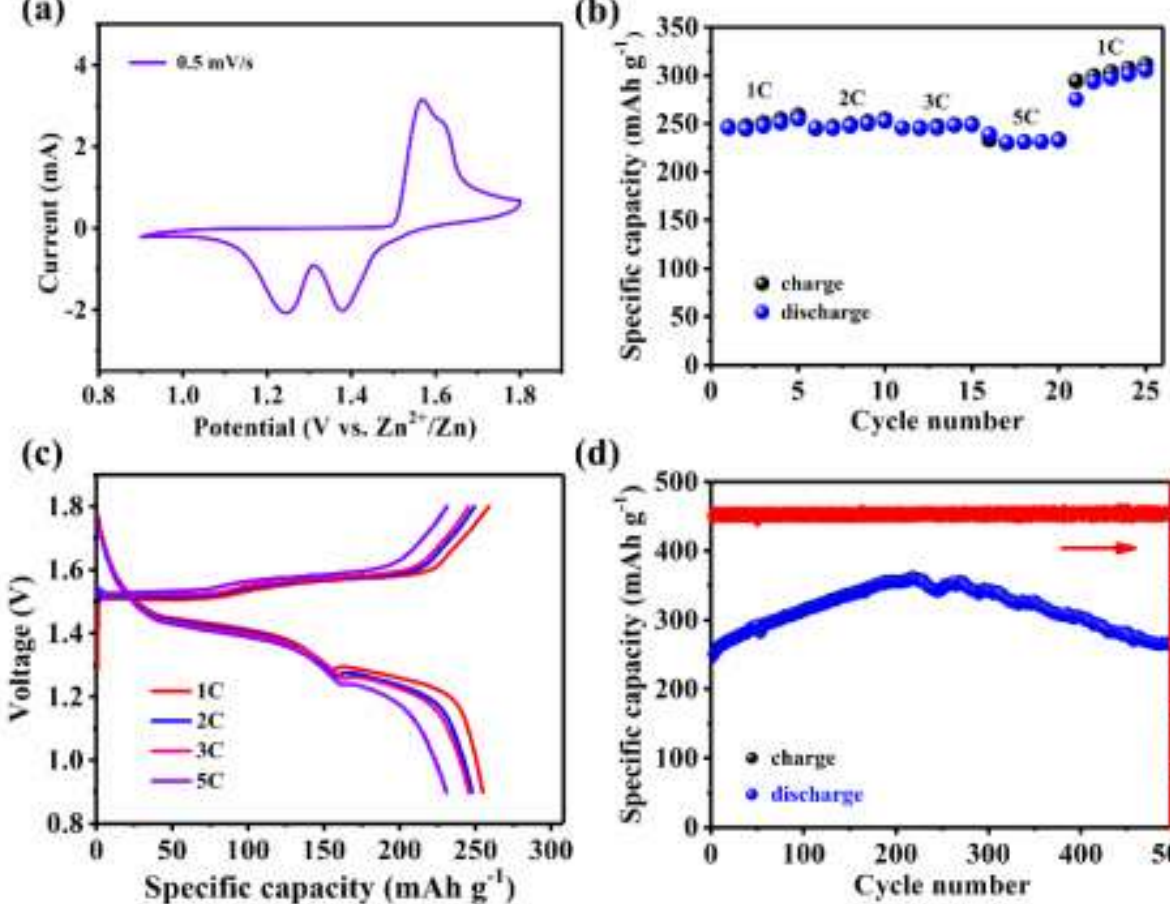

(d)

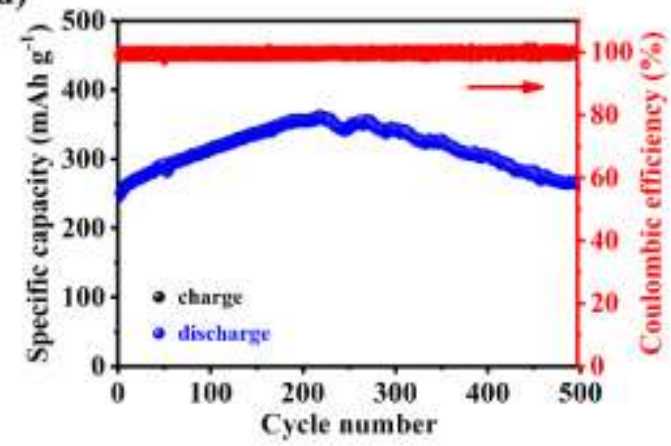

Figure S14. (a) CV curve of the coin ZIBs at $0.5 \mathrm{mV} / \mathrm{s}$, (b) Rate capacity of the coin

ZIBs at different rates and (c) the corresponding charge-discharge curves, (d) Long term cycle performance of the coin ZIBs at $4 \mathrm{C}$.

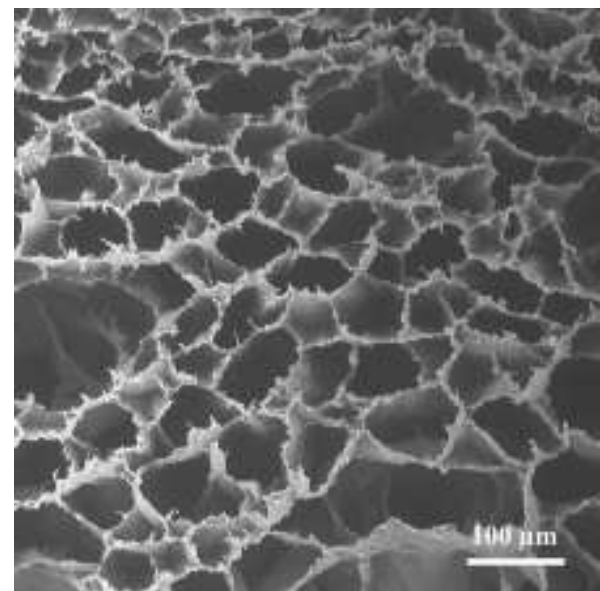

Figure S15. SEM image of XG-PAM/CNF hydrogel after 1000 cycles. 
(a)

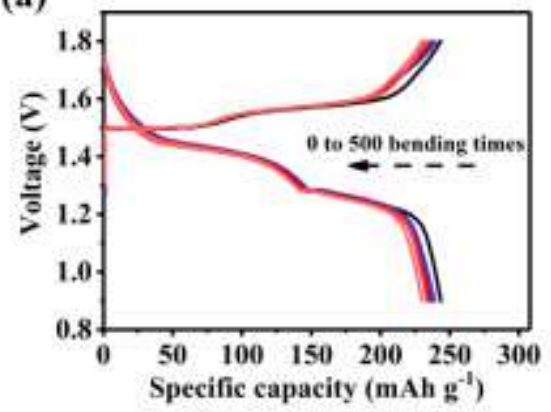

(c)

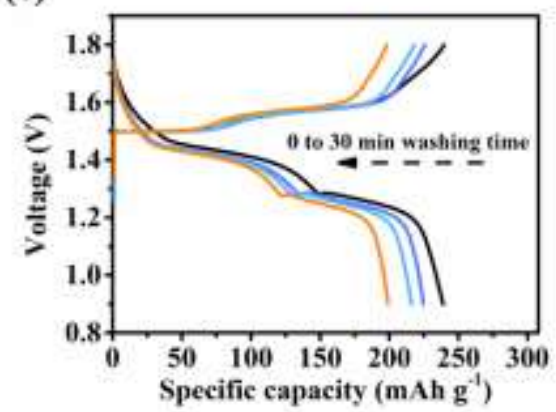

(b)

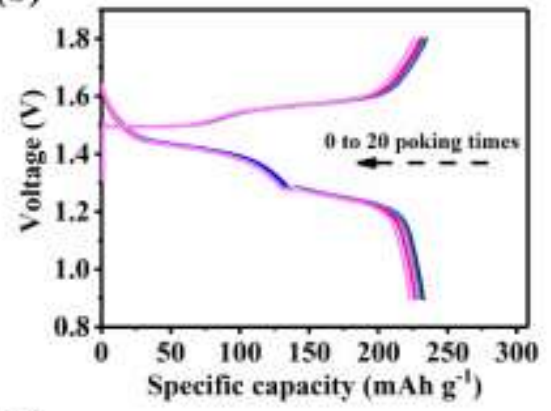

(d)

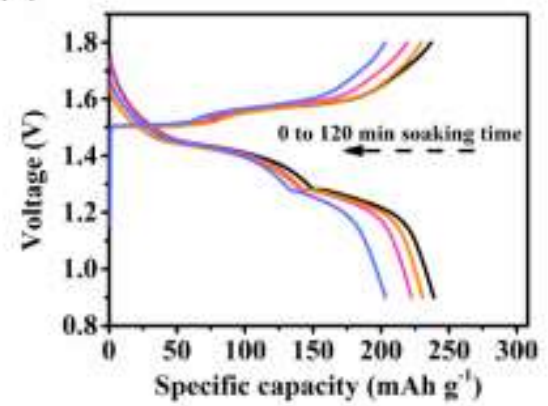

Figure S16. Charge-discharge curves of the flexible ZIBs under severe tests. (a)

Bending test, (b) Poking test, (c) Washing test, (d) Soaking test.

(a)

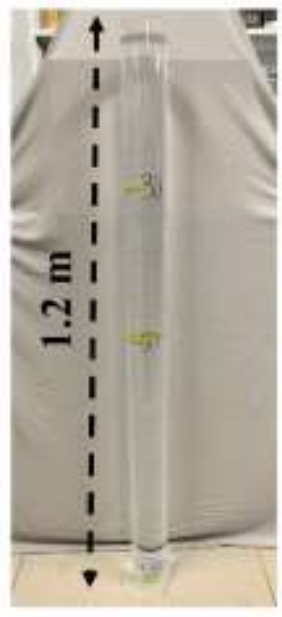

(b)

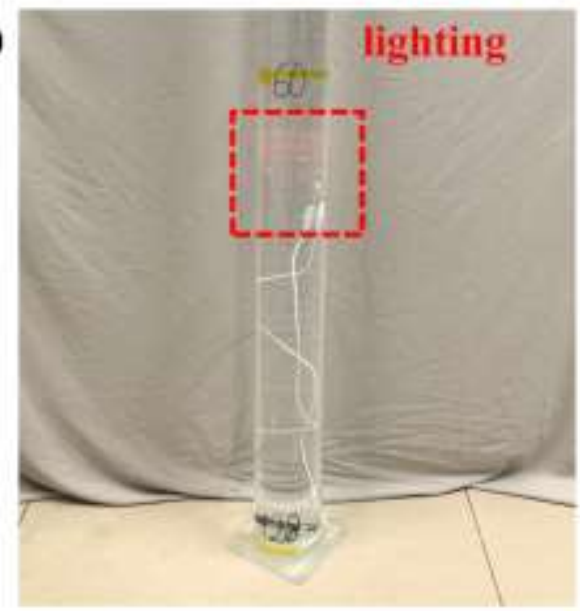

Figure S17. (a) A 1.2-meter-long acrylic cylinder filled with deionized water, (b) Unpackaged flexible ZIBs achieved lighting up a red LED light at the depth of 1.2 meters. 
Table S1 The process of calculating the ionic conductivity of hydrogel electrolytes

\begin{tabular}{ccccc}
\hline Hydrogel electrolytes & $\mathbf{L}$ & $\mathbf{A}$ & $\mathbf{R}$ & $\boldsymbol{\sigma}$ \\
& $(\mathbf{c m})$ & $\left(\mathbf{c m}^{2}\right)$ & $(\boldsymbol{\Omega})$ & $\left(\mathbf{m S ~ c m}^{-\mathbf{1}}, \boldsymbol{\sigma}=\mathbf{L} / \mathbf{( R \cdot A ) \times \mathbf { 1 0 0 0 } )}\right.$ \\
\hline PAM & 0.025 & 6.0 & 0.311 & 13.4 \\
PAM/CNF & 0.025 & 6.0 & 0.205 & 20.3 \\
XG-PAM/CNF & 0.025 & 6.0 & 0.145 & 28.8 \\
\hline
\end{tabular}

Table S2 Summary of recent reported flexible ZIBs based on hydrogel electrolytes

\begin{tabular}{|c|c|c|c|c|c|}
\hline Cathode & Anode & Electrolyte & 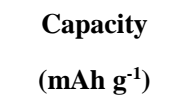 & Cycling performance & Reference \\
\hline $\begin{array}{c}\mathrm{MnO}_{2} @ \mathrm{CNT} \\
\text { paper }\end{array}$ & $\begin{array}{l}\text { Deposited-Zn } \\
@ \text { CNT paper }\end{array}$ & $\begin{array}{c}\mathrm{ZnSO}_{4} / \mathrm{MnSO}_{4} / \mathrm{Zn}- \\
\text { alginate/PAAm } \\
\text { hydrogel }\end{array}$ & $\begin{array}{c}300.4 \\
\left(110 \mathrm{~mA} \mathrm{~g}^{-1}\right)\end{array}$ & $\begin{array}{c}82 \% \text { capacity } \\
\text { retention after } 500 \\
\text { cycles }\left(880 \mathrm{~mA} \mathrm{~g}^{-1}\right)\end{array}$ & 2 \\
\hline $\begin{array}{c}\mathrm{MnO}_{2} / \mathrm{CNT} @ \\
\mathrm{CNT} \text { paper }\end{array}$ & $\begin{array}{l}\text { Deposited-Zn } \\
@ \text { CNT paper }\end{array}$ & $\begin{array}{l}\mathrm{ZnSO}_{4} / \mathrm{MnSO}_{4} / \\
\text { gelatin-g-PAM }\end{array}$ & $\begin{array}{c}282 \\
\left(308 \mathrm{~mA} \mathrm{~g}^{-1}\right)\end{array}$ & $\begin{array}{l}97 \% \text { capacity } \\
\text { retention after } 1000 \\
\text { cycles }\left(2772 \mathrm{~mA} \mathrm{~g}^{-}\right. \\
1)\end{array}$ & 3 \\
\hline $\begin{array}{l}\mathrm{MnO}_{2} / \mathrm{CNT} @ \\
\text { carbon paper }\end{array}$ & $\mathrm{Zn}$ foil & $\begin{array}{c}\mathrm{ZnSO}_{4} / \mathrm{PSBMA} \\
\text { hydrogel }\end{array}$ & $\begin{array}{c}150 \\
\left(500 \mathrm{~mA} \mathrm{~g}^{-1}\right)\end{array}$ & $\begin{array}{l}67 \% \text { capacity } \\
\text { retention after } 100 \\
\text { cycles }\left(500 \mathrm{~mA} \mathrm{~g}^{-1}\right)\end{array}$ & 4 \\
\hline $\begin{array}{c}\mathrm{MnO}_{2} @ \mathrm{PEDOT} \\
@ \mathrm{CC}\end{array}$ & $\begin{array}{l}\text { Deposited- } \\
\text { Zn@CC }\end{array}$ & $\begin{array}{c}\mathrm{ZnCl}_{2} / \mathrm{MnSO}_{4} / \\
\text { PVA gel }\end{array}$ & $\begin{array}{c}282.4 \\
\left(700 \mathrm{~mA} \mathrm{~g}^{-1}\right)\end{array}$ & $\begin{array}{l}77.7 \% \text { capacity } \\
\text { retention after } 300 \\
\text { cycles }\left(1860 \mathrm{~mA} \mathrm{~g}^{-}\right. \\
1)\end{array}$ & 5 \\
\hline $\mathrm{MnO}_{2} @ \mathrm{CC}$ & $\begin{array}{l}\text { Deposited- } \\
\text { Zn@CC }\end{array}$ & $\begin{array}{c}\mathrm{ZnSO}_{4} / \mathrm{MnSO}_{4} / \\
\text { gelatin gel }\end{array}$ & $\begin{array}{l}265 \\
(1 \mathrm{C})\end{array}$ & $\begin{array}{c}76.9 \% \text { capacity } \\
\text { retention after } 1000 \\
\text { cycles }(4 \mathrm{C})\end{array}$ & 6 \\
\hline $\begin{array}{c}\mathrm{MnO}_{2} @ \text { carbon } \\
\text { paper }\end{array}$ & Zn plate & $\begin{array}{c}\mathrm{Zn}\left(\mathrm{NO}_{3}\right)_{2} / \mathrm{MnSO}_{4} / \\
\mathrm{PVA}-\mathrm{COOH} \\
\text { hydrogel }\end{array}$ & $\begin{array}{l}216.5 \\
(1 \mathrm{C})\end{array}$ & $\begin{array}{c}83 \% \text { capacity } \\
\text { retention after } 1000 \\
\text { cycles }(1 \mathrm{C})\end{array}$ & 7 \\
\hline $\begin{array}{c}\text { Deposited } \\
\mathrm{MnO}_{2} @ \mathrm{CNT} \\
\text { film }\end{array}$ & $\mathrm{Zn}$ foil & $\begin{array}{c}\mathrm{ZnSO}_{4} / \mathrm{MnSO}_{4} / \\
\text { xanthan gum } \\
\text { hydrogel }\end{array}$ & $\begin{array}{l}260 \\
(1 \mathrm{C})\end{array}$ & $\begin{array}{c}90 \% \text { capacity } \\
\text { retention after } 330 \\
\text { cycles }(1 \mathrm{C})\end{array}$ & 8 \\
\hline $\mathrm{MnO}_{2} / \mathrm{CNT} @ \mathrm{CC}$ & $\begin{array}{l}\text { Deposited- } \\
\text { Zn@CC }\end{array}$ & $\begin{array}{l}\mathrm{ZnSO}_{4} / \mathrm{MnSO}_{4} / \\
\mathrm{XG}-\mathrm{PAM} / \mathrm{CNF} \\
\text { hydrogel }\end{array}$ & $\begin{array}{l}237 \\
(1 \mathrm{C})\end{array}$ & $\begin{array}{c}86.2 \% \text { capacity } \\
\text { retention after } 1000 \\
\text { cycles }(4 \mathrm{C})\end{array}$ & $\begin{array}{l}\text { This } \\
\text { work }\end{array}$ \\
\hline
\end{tabular}


Table S3 Summary of performance in harsh conditions for recent reported flexible ZIBs.

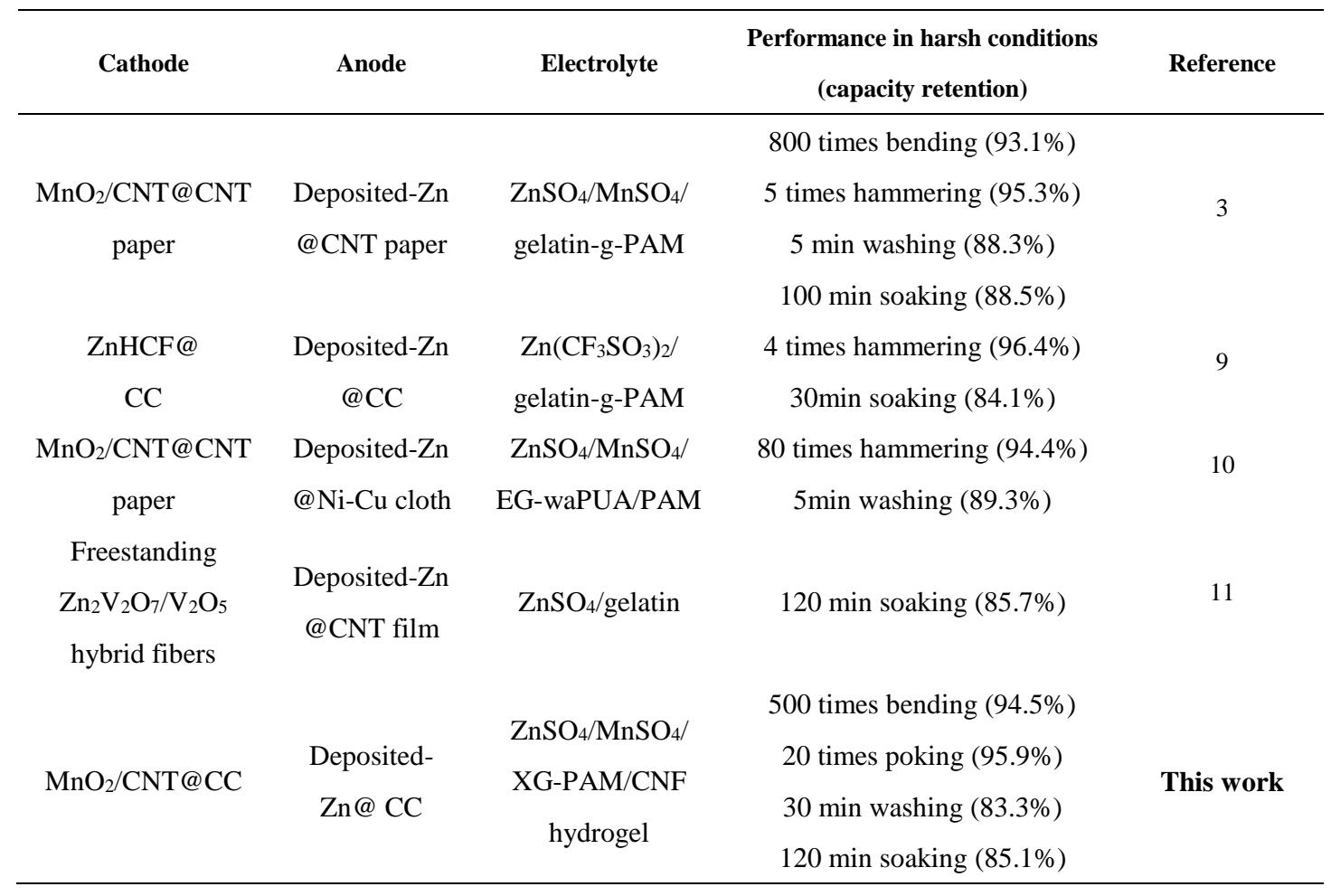




\section{Supporting References}

(1) Zhu, M.; Wang, X.; Tang, H.; Wang, J.; Hao, Q.; Liu, L.; Li, Y.; Zhang, K.; Schmidt, O. G. Antifreezing Hydrogel with High Zinc Reversibility for Flexible and Durable Aqueous Batteries by Cooperative Hydrated Cations. Adv. Funct. Mater. 2019, 1907218.

(2) Liu, Z.; Wang, D.; Tang, Z.; Liang, G.; Yang, Q.; Li, H.; Ma, L.; Mo, F.; Zhi, C. A Mechanically Durable and Device-Level Tough $\mathrm{Zn}-\mathrm{MnO}_{2}$ Battery with High Flexibility. Energy Storage Mater. 2019, 23, 636-645.

(3) Li, H.; Han, C.; Huang, Y.; Huang, Y.; Zhu, M.; Pei, Z.; Xue, Q.; Wang, Z.; Liu, Z.; Tang, Z.; Wang, Y.; Kang, F.; Li, B.; Zhi, C. An Extremely Safe and Wearable Solid-State Zinc Ion Battery Based on a Hierarchical Structured Polymer Electrolyte. Energy Environ. Sci. 2018, 11 (4), 941-951.

(4) Leng, K.; Li, G.; Guo, J.; Zhang, X.; Wang, A.; Liu, X.; Luo, J. A Safe Polyzwitterionic Hydrogel Electrolyte for Long-Life Quasi-Solid State Zinc Metal Batteries. Adv. Funct. Mater. 2020, 30 (23), 2001317.

(5) Zeng, Y.; Zhang, X.; Meng, Y.; Yu, M.; Yi, J.; Wu, Y.; Lu, X.; Tong, Y. Achieving Ultrahigh Energy Density and Long Durability in a Flexible Rechargeable Quasi-Solid-State $\mathrm{Zn}-\mathrm{MnO}_{2}$ Battery. Adv. Mater. 2017, 29 (26), 1700274. 
(6) Wang, Z.; Ruan, Z.; Ng, W. S.; Li, H.; Tang, Z.; Liu, Z.; Wang, Y.; Hu, H.; Zhi, C. Integrating a Triboelectric Nanogenerator and a Zinc-Ion Battery on a Designed Flexible 3D Spacer Fabric. Small Methods 2018, 2 (10), 1800150.

(7) Li, Q.; Cui, X.; Pan, Q. Self-Healable Hydrogel Electrolyte toward HighPerformance and Reliable Quasi-Solid-State $\mathrm{Zn}-\mathrm{MnO}_{2}$ Batteries. ACS Appl. Mater. Interfaces 2019, 11 (42), 38762-38770.

(8) Zhang, S.; Yu, N.; Zeng, S.; Zhou, S.; Chen, M.; Di, J.; Li, Q. An Adaptive and Stable Bio-Electrolyte for Rechargeable Zn-Ion Batteries. J. Mater. Chem. A 2018, $6(26), 12237-12243$.

(9) Chen, Z.; Wang, P.; Ji, Z.; Wang, H.; Liu, J.; Wang, J.; Hu, M.; Huang, Y. HighVoltage Flexible Aqueous Zn-Ion Battery with Extremely Low Dropout Voltage and Super-Flat Platform. Nano-Micro Lett. 2020, 12 (1), 75.

(10) Mo, F.; Liang, G.; Meng, Q.; Liu, Z.; Li, H.; Fan, J.; Zhi, C. A Flexible Rechargeable Aqueous Zinc Manganese-Dioxide Battery Working at $-20{ }^{\circ} \mathrm{C}$. Energy Environ. Sci. 2019, 12 (2), 706-715.

(11) Wang, H.; Zhang, S.; Deng, C. In Situ Encapsulating Metal Oxides into CoreShell Hierarchical Hybrid Fibers for Flexible Zinc-Ion Batteries toward High Durability and Ultrafast Capability for Wearable Applications. ACS Appl. Mater. Interfaces 2019, 11 (39), 35796-35808. 